\title{
Poster: Combinatorics, Algorithms and Systems for Sensor Deployment With Line-Of-Sight Constraints
}

\author{
Kin Sum Liu \\ Stony Brook University \\ kiliu@cs.stonybrook.edu
}

\author{
Brent Schiller \\ Stony Brook University \\ brent.schiller@stonybrook.jgao@cs.stonybrook.edu \\ edu
}

\author{
Shan Lin \\ Stony Brook University \\ shan.x.lin@stonybrook.edu js
}

\author{
Joseph S. B. Mitchell \\ Stony Brook University \\ jsbm@ams.sunysb.edu
}

\begin{abstract}
In this paper we investigate sensor deployment and coverage algorithms for using infrared signals in indoor applications. Infrared signals are directional and reliable signals that have little interference with other electromagnetic signals that are commonly found in the deployment domain such as visible light and wireless radio waves. Since the angle of arrival is used, and line of sight is the main constraint for IR signals, we investigate the problem called robust guarding, i.e., placing emitters to ensure that all points of the domain are robustly covered by two emitters that are from sufficiently different directions. We prove combinatorial upper and lower bounds for the number of emitters needed and prove that finding the minimum number of guards is NP-hard. We show that $n / 2$ guards are always sufficient and sometimes necessary for rectilinear polygons and we provide practical algorithms in general. We also developed a testbed with low cost off-the-shelf infrared (IR) emitters and sensors for indoor device-free localization. We tested the algorithms for using infrared sensors for indoor localization and our system achieves an average accuracy of $11.7 \mathrm{~cm}$ in a typical office setting.
\end{abstract}

\section{INTRODUCTION}

In this paper we study the problem of sensor deployment and coverage when the coverage range is largely influenced by line of sight constraints. Our problem is strongly motivated by the increasing interest in exploring signals other than RFs, cameras, and ultrasound for use in a variety of indoor applications.

In using infrared for localization a target's location is determined when emitters generate infrared signals while infrared sensors capture the shadows caused by the targets and/or the reflections from the targets. Angles of arrival are derived from the angle of the emitter to the target. By

Permission to make digital or hard copies of part or all of this work for personal or classroom use is granted without fee provided that copies are not made or distributed for profit or commercial advantage and that copies bear this notice and the full citation on the first page. Copyrights for third-party components of this work must be honored. For all other uses, contact the owner/author(s).

MobiHoc'16 July 04-08, 2016, Paderborn, Germany

(C) 2016 Copyright held by the owner/author(s).

ACM ISBN 978-1-4503-4184-4/16/07.

DOI: http://dx.doi.org/10.1145/2942358.2947403 using two such angles of arrival from different emitters the target's location can theoretically be determined. In practice however, the deployment of emitters is non-trivial. Since infrared propagates in a directional manner, careful placement of emitters with respect to the building layout is required to achieve full coverage in a complex indoor environment. In addition, since the angle of arrival is used for localization, having two emitters in the same direction will lead to a large overlap in the shadow that they cast which could substantially reduce the localization accuracy.

In this paper, we investigate the deployment problem for using infrared-based indoor localization. We place emitters as guards and require each point $p$ of the polygon to be $\alpha$ robustly guarded, in the sense that $p$ is visible to at least two guards (namely, the emitters) with an angle of separation of at least $\alpha, 0<\alpha \leq \pi / 2$. We show that finding a minimum number of guards to $\alpha$-robustly guard a simple polygon is NP-hard. And we present both combinatorial bounds and algorithms. For a general polygon of $n$ vertices, $\lfloor 3 n / 4\rfloor$ guards are sometimes necessary (while for a standard art gallery problem $\lfloor n / 3\rfloor$ guards are both sufficient and sometimes necessary). For rectilinear polygons and placements of guards on the vertices, we show that $\lfloor n / 2\rfloor$ guards are sometimes necessary, and surprisingly, always sufficient we prove that placing guards in alternating order along the polygon boundary is an $\alpha$-robust guarding solution. This provides not only tight bounds for robust guarding in rectilinear cases but also a practical algorithm for sensor deployment in an indoor setting when the deployment region can be approximated by a rectilinear polygon.

We develop algorithms for both single target and multiple target detection using infrared sensors. To test the algorithms, we developed customized Infrared Localization nodes, called iLoc nodes, with off-the-shelf infrared (IR) emitters and sensors with component costs of only a few dollars. There are two types of iLoc nodes: iLoc guard nodes and iLoc strip nodes, as shown in Figure 1. The iLoc guard node actively generates omni-directional IR beams through a customized emitter array. It has an (approximately) 170degree divergence angle and a reasonably long effective sensing range up to $23 \mathrm{~m}$.

An array of angled IR sensors is installed along with the emitters on the iLoc guard. They are used for detecting reflections caused by the presence of a nearby target. The iLoc strip node has an IR sensor array which is used in 
conjunction with the iLoc guard to detect IR shadows in the deployed environment. It is thin and long so that it can be easily deployed on the wall in an indoor environment. Multiple iLoc guards and strip nodes deployed at the same location are synchronized over wireless channels to perform real-time localization and tracking.

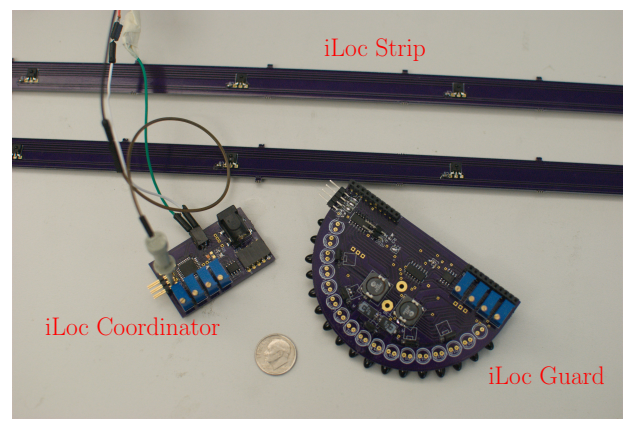

Figure 1: iLoc Components

The iLoc localization system was tested in different indoor environments. Experimental results show that our design detects human location in an office setting in real-time with an average error of $11.7 \mathrm{~cm}$ which outperforms many other device-free techniques. The contributing factors for this high precision localization are the accurate detection of shadows and reflections by the new hardware design, the collaborative sensing of the localization scheme, and our guard deployment algorithm.

\section{ROBUST GUARDING: COMBINATORICS AND ALGORITHMS}

To achieve full coverage in an indoor environment, we need to deploy the iLoc devices effectively and efficiently. We model the sensor deployment and its quality requirement as the robust guarding problem: the indoor environment is regarded as a polygon and iLoc guard nodes as point guards. iLoc strips of sensors are deployed along the boundary of this polygon to detect the arrival angle for localization.

An $\alpha$-robust guarding solution for a polygon $P$ requires that each point $p$ of $P$ is visible to at least two guards $g_{1}, g_{2}$ such that the angle between the two rays $p g_{1}$ and $p g_{2}$ is at least $\alpha$. We provide an upper bounds on the number of guards required as well as algorithms for guard placement in any rectilinear polygon and a lower bound for the worst case. We focus on the case of placing guards only at vertices of $P$ and we consider the case when $\alpha \in(0, \pi / 2]$.

Theorem 2.1. Let $P$ be a simple polygon with $n$ vertices. Finding an optimal set of guards to $\alpha$-robustly guard every point $p \in P$ is NP-hard.

Theorem 2.2. Let $P$ be a simple polygon with $n$ vertices. $\lfloor 3 n / 4\rfloor+1$ vertex guards are sometimes necessary to $\alpha$ robustly guard $P$.

Theorem 2.3. Let $P$ be a simple rectilinear polygon with $n$ vertices. Then, $n / 2$ vertex guards are always sufficient and sometimes necessary to $\alpha$-robustly guard $P$.

\section{LOCALIZATION}

For our system, $e$ emitters take turn emitting light in a predefined sequence for $t$ seconds. As an example, when the $i$-th emitter is emitting an infrared pulse, the $j$-th strip sensor processes the signal and reports a probability $p_{\text {shadow }}^{j}$. For reflection, the angled sensors on the emitter report a probability $p_{\text {reflection }}^{i}$ with a distance $d_{i}$. Then we can define a metric for the estimated location $(x, y)$ for the object based on the IR pulse emitted by the $i$-th emitter as

$$
\begin{aligned}
L^{i}(x, y)= & \frac{1}{\sum_{k=1}^{r}\left\lceil p_{\text {shadow }}^{k}\right\rceil} \times \sum_{j=1}^{r}\left(p_{\text {shadow }}^{j} \times\right. \\
& \left.\frac{\left|\left(y_{j}-y_{i}\right) x-\left(x_{j}-x_{i}\right) y+x_{j} y_{i}-y_{j} x_{i}\right|}{\sqrt{\left(y_{j}-y_{i}\right)^{2}+\left(x_{j}-x_{i}\right)^{2}}}\right)+ \\
& p_{\text {reflection }}^{i} \times\left|\sqrt{\left(x-x_{i}\right)^{2}+\left(y-y_{i}\right)^{2}}-d_{i}\right|
\end{aligned}
$$

Then, to integrate the signals from different emitters, we minimize these metrics for all $e$ emitters together so that the estimated location aligns with the signals in the whole sensing cycle as much as possible:

$$
\left(x^{*}, y^{*}\right)=\underset{x, y}{\arg \min } \sum_{i=1}^{e} L^{i}(x, y)
$$

After the minimization, $\left(x^{*}, y^{*}\right)$ is our best estimation for the object.

\section{EVALUATION}

Localization experiments have been conducted to locate a human in an office room of $409 \mathrm{~cm}$ by $255 \mathrm{~cm}$. The room was visualized by the boundary of polygon in Figure 2. Since it is a polygon with 6 vertices, we placed iLoc guard nodes at vertices according to Theorem 2.3. iLoc sensor strips were deployed on all walls. For each of the 16 locations, a snapshot of the readings was taken and fed in real time to the localization program.

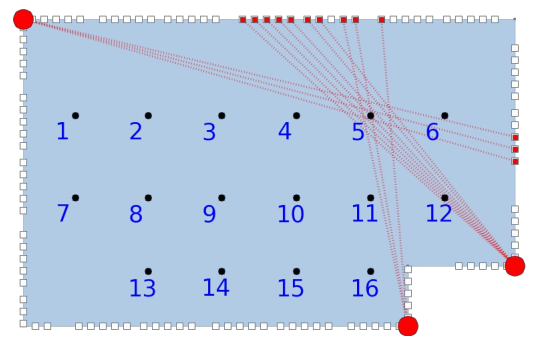

Figure 2: Testbed Layout. The three red dots at vertices represent iLoc guard nodes; the white dots along the edges represent iLoc sensors; the black dots represent the testing locations. This figure demonstrates the case when a person is at location 5 .

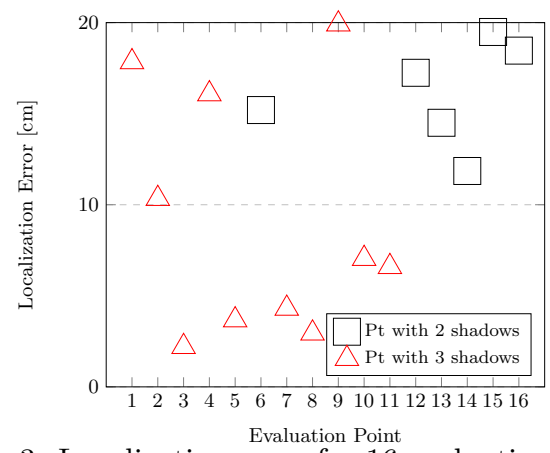

Figure 3: Localization error for 16 evaluation points 